\title{
FLOW INVARIANCE FOR PERTURBED NONLINEAR EVOLUTION EQUATIONS
}

\author{
DIETER BOTHE
}

\begin{abstract}
Let $X$ be a real Banach space, $J=[0, a] \subset \mathbb{R}, A: D(A) \subset$ $X \rightarrow 2^{X} \backslash \emptyset$ an $m$-accretive operator and $f: J \times X \rightarrow X$ continuous. In this paper we obtain necessary and sufficient conditions for weak positive invariance (also called viability) of closed sets $K \subset X$ for the evolution system

$$
u^{\prime}+A u \ni f(t, u) \text { on } J=[0, a] .
$$

More generally, we provide conditions under which this evolution system has mild solutions satisfying time-dependent constraints $u(t) \in K(t)$ on $J$. This result is then applied to obtain global solutions of reaction-diffusion systems with nonlinear diffusion, e.g. of type

$$
u_{t}=\Delta \Phi(u)+g(u) \text { in }(0, \infty) \times \Omega,\left.\quad \Phi(u(t, \cdot))\right|_{\partial \Omega}=0, u(0, \cdot)=u_{0}
$$

under certain assumptions on the set $\Omega \subset \mathbb{R}^{n}$ the function $\Phi\left(u_{1}, \ldots, u_{m}\right)=$ $\left(\varphi_{1}\left(u_{1}\right), \ldots, \varphi_{m}\left(u_{m}\right)\right)$ and $g: \mathbb{R}_{+}^{m} \rightarrow \mathbb{R}^{m}$.
\end{abstract}

\section{INTRODUCTION}

Let $X$ be a real Banach space and $A: D(A) \subset X \rightarrow 2^{X} \backslash \emptyset m$-accretive, where $2^{X} \backslash \emptyset$ denotes the nonempty subsets of $X$. Given $K: J=[0, a] \rightarrow$ $2^{X} \backslash \emptyset$ with closed values $K(t)$ such that $K_{A}(t):=K(t) \cap \overline{D(A)} \neq \emptyset$ on $J$ and a continuous $f: \operatorname{gr}\left(K_{A}\right) \rightarrow X$, we consider the initial value problem

$$
u^{\prime}+A u \ni f(t, u) \quad \text { on } J, \quad u(0)=x_{0} .
$$

Given any initial value $x_{0} \in K_{A}(0)$, we look for a mild solution $u$ of (1), by which we mean a continuous $u: J \rightarrow X$ such that $u$ is the mild solution of the quasi-autonomous problem

$$
u^{\prime}+A u \ni w(t) \quad \text { on } J, \quad u(0)=x_{0},
$$

1991 Mathematics Subject Classification. 34G20, 35K57.

Key words and phrases. Nonlinear evolution equation, time-dependent constraints, viability, reaction-diffusion system, global existence.

The author gratefully acknowledges financial support by DAAD within the scope of the French-German project PROCOPE.

Received: November 22, 1996. 
with $w(t)=f(t, u(t))$ on $J$; notice that $u$ then automatically has to satisfy $u(t) \in K_{A}(t)$ on $J$, since $f$ is only defined on $\operatorname{gr}\left(K_{A}\right)$.

Suppose that (1) has mild solution $u$ and let $v$ be the mild solution of

$$
v^{\prime}+A v \ni f\left(0, x_{0}\right) \quad \text { on } J, \quad v(0)=x_{0} .
$$

By continuity of $f$ and $u$ it follows that

$$
\frac{1}{h}|u(h)-v(h)| \leq \frac{1}{h} \int_{0}^{h}\left|f(t, u(t))-f\left(0, x_{0}\right)\right| d t \rightarrow 0 \quad \text { as } h \rightarrow 0+,
$$

hence

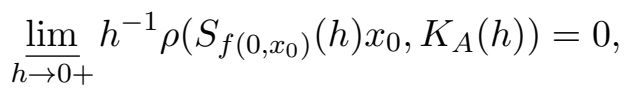

where $S_{z}(\cdot)$ denotes the semigroup generated by $-A_{z}$ with $A_{z} x:=A x-z$ on $D\left(A_{z}\right)=D(A)$.

By weak positive invariance of $K(\cdot)$ for $u^{\prime}+A u \ni f(t, u)$ we mean that (1) has a mild solution on $J_{\tau}=[\tau, a]$ for every $\tau \in[0, a)$ and every initial value $u(\tau)=x_{0} \in K_{A}(\tau)$. The argument given above shows that

$$
f(t, x) \in T_{K}^{A}(t, x) \quad \text { for all }(t, x) \in \operatorname{gr}\left(K_{A}\right) \text { with } t<a
$$

is a necessary condition for weak positive invariance of $K(\cdot)$, where $T_{K}^{A}$ is defined on $\operatorname{gr}\left(K_{A}\right) \cap([0, a) \times X)$ by

$$
T_{K}^{A}(t, x)=\left\{z \in X: \lim _{h \rightarrow 0+} h^{-1} \rho\left(S_{z}(h) x, K_{A}(t+h)\right)=0\right\} .
$$

In the special case $A=0$ this becomes

$$
T_{K}(t, x)=\left\{z \in X: \underline{\lim _{h \rightarrow 0+}} h^{-1} \rho(x+h z, K(t+h))=0\right\},
$$

and if, in addition, $K(t) \equiv K$ holds then $T_{K}(t, x)=T_{K}(x)$ is the Bouligand contingent cone w.r. to $K$ at the point $x$; see e.g. $\S 4.1$ in [10].

Since all $K(t)$ are closed by assumption, it is also natural to assume that $\operatorname{gr}\left(K_{A}\right)$ is closed from the left, i.e.

$\left(t_{n}\right) \subset J$ with $t_{n} \nearrow t$ and $x_{n} \in K_{A}\left(t_{n}\right)$ with $x_{n} \rightarrow x$ implies $x \in K_{A}(t)$;

notice that if there are mild solutions $u_{n}$ with $u_{n}\left(t_{n}\right)=x_{n}$, then $K_{A}(t) \ni$ $u_{n}(t) \rightarrow x$.

In this situation we will show that the "subtangential condition" (2) is also sufficient, provided the semigroup generated by $-A$ is compact and $f$ satisfies the growth condition

$$
|f(t, x)| \leq c(1+|x|) \text { on } \operatorname{gr}\left(K_{A}\right) \text { with some } c>0 .
$$

In the final section this result is applied to a class of RD-systems including the model problem mentioned in the abstract above, and sufficient conditions for existence of global solutions are obtained. 


\section{Preliminaries}

Let $X$ be a real Banach space with norm $|\cdot|$. Then $\bar{B}_{r}(x)$ denotes the closed ball in $X$ with center $x$ and radius $r, B_{r}(x)$ its interior and $\rho(x, B)$ is the distance from $x$ to the set $B \subset X$, with the usual convention $\rho(x, \emptyset)=\infty$. Given $J=[0, a] \subset \mathbb{R}$, we let $C_{\mathrm{X}}(J)$ be the Banach space of all continuous $u: J \rightarrow X$ and $L_{\mathrm{X}}^{1}(J)$ the Banach space of all equivalence classes (w.r. to equality a.e.) of strongly measurable, Bochner-integrable $w: J \rightarrow X$, both equipped with the usual norms which we denote by $|\cdot|_{0}$, respectively $|\cdot|_{1}$. Given an operator $A: X \rightarrow 2^{X}$, we let $D(A)=\{x \in X: A x \neq \emptyset\}$, $R(A)=\bigcup_{x \in D(A)} A x$ and $\operatorname{gr}(A)=\{(x, y): x \in D(A), y \in A x\}$ denote the domain, range and graph of $A$, respectively.

Recall that $A: X \rightarrow 2^{X}$ is $m$-accretive if $R(I+\lambda A)=X$ for all $\lambda>0$ and $A$ is accretive, which means

$$
(y-\bar{y}, x-\bar{x})_{+} \geq 0 \quad \text { for all } x, \bar{x} \in D(A), y \in A x \text { and } \bar{y} \in A \bar{x} .
$$

Here $(\cdot, \cdot)_{+}$is given by $(z, x)_{+}=\max \left\{x^{*}(z): x^{*} \in \mathcal{F}(x)\right\}$ where $\mathcal{F}: X \rightarrow$ $2^{X^{*}} \backslash \emptyset$ denotes the duality map, i.e. $\mathcal{F}(x)=\left\{x^{*} \in X^{*}: x^{*}(x)=|x|^{2}=\right.$ $\left.\left|x^{*}\right|^{2}\right\}$; see e.g. $\S 12.2$ in [9].

If $A$ is $m$-accretive, the resolvents $J_{\lambda}:=(I+\lambda A)^{-1}: X \rightarrow D(A)$ are nonexpansive mappings, i.e. $\left|J_{\lambda} x-J_{\lambda} y\right| \leq|x-y|$ on $X \times X$, for all $\lambda>0$. Given $x \in D(A)$ we have $\left|J_{\lambda} x-x\right| \leq \lambda|y|$ for $\lambda>0$, where $y$ is any element of $A x$, which implies $J_{\lambda} x \rightarrow x$ as $\lambda \rightarrow 0+$ on $\overline{D(A)}$. The resolvents satisfy the so-called resolvent identity

$$
J_{\lambda} x=J_{\mu}\left(\frac{\mu}{\lambda} x+\frac{\lambda-\mu}{\lambda} J_{\lambda} x\right) \quad \text { on } X \text { for all } \lambda, \mu>0 .
$$

If $A$ is $m$-accretive, it generates a semigroup $\{S(t)\}_{t \geq 0}$ of nonexpansive mappings $S(t): \overline{D(A)} \rightarrow \overline{D(A)}$, given by the so-called exponential formula, i.e.

$$
S(t) x=\lim _{n \rightarrow \infty} J_{t / n}^{n} x \quad \text { for } t \geq 0 \text { and } x \in \overline{D(A)} .
$$

Then $\{S(t)\}_{t \geq 0}$ is called the semigroup generated by $-A$, and it is said to be compact if $\overline{S(t) B}$ is compact for all $t>0$ and bounded $B \subset \overline{D(A)}$ (i.e. the $S(t)$ are compact maps for $t>0)$. Let us note in passing that $\{S(t)\}_{t \geq 0}$ is compact iff $\{S(t)\}_{t>0}$ is equicontinuous and $J_{\lambda}$ is a compact map for some (or, equivalently, for all) $\lambda>0$.

Let us also recall some facts concerning the quasi-autonomous problem

$$
u^{\prime}+A u \ni w(t) \quad \text { on } J_{\tau}=[\tau, a], \quad u(\tau)=x_{0},
$$

where $\tau \in[0, a)$. For $m$-accretive $A$, given any $w \in L_{\mathrm{X}}^{1}\left(J_{\tau}\right)$ and $x_{0} \in \overline{D(A)}$, the initial value problem (4) has a unique mild solution $u$. This means that $u: J_{\tau} \rightarrow \overline{D(A)}$ is continuous with $u(\tau)=x_{0}$ and $u$ is the uniform limit of $\epsilon$-DS-approximate solutions $u^{\epsilon}$ as $\epsilon \rightarrow 0+$. Here, by an $\epsilon$-DS-approximate solution $u^{\epsilon}$ of (4) one means a function $u^{\epsilon}$ with $u^{\epsilon}\left(t_{0}\right)=x_{0}$ and $u^{\epsilon}(t)=x_{k}$ 
on $\left(t_{k-1}, t_{k}\right]$ for $k=1, \ldots, m$, where $\tau=t_{0}<t_{1}<\cdots<t_{m}<a \leq t_{m}+\epsilon$ with $t_{k}-t_{k-1} \leq \epsilon$ and the $x_{k}$ solve the implicit difference scheme

$$
\frac{x_{k}-x_{k-1}}{t_{k}-t_{k-1}}+A x_{k} \ni z_{k} \quad \text { for } k=1, \ldots, m
$$

with $z_{1}, \ldots, z_{m} \in X$ such that $\sum_{k=1}^{m} \int_{t_{k-1}}^{t_{k}}\left|z_{k}-w(t)\right| d t \leq \epsilon$.

In fact, every sequence of such $\epsilon_{m}$-DS-approximate solutions $u^{\epsilon_{m}}$ converges to $u$ uniformly on $[\tau, a)$ if $\epsilon_{m} \rightarrow 0+$.

In the sequel $u\left(\cdot ; \tau, x_{0}, w\right)$ denotes the mild solution of (4) and we shall use the following property: If $w, \bar{w} \in L_{\mathrm{X}}^{1}\left(J_{\tau}\right)$ and $x_{0}, \bar{x}_{0} \in \overline{D(A)}$ then

$$
\left|u\left(t ; \tau, x_{0}, w\right)-u\left(t ; \tau, \bar{x}_{0}, \bar{w}\right)\right| \leq\left|x_{0}-\bar{x}_{0}\right|+\int_{\tau}^{t}|w(s)-\bar{w}(s)| d s \quad \text { for } t \in J_{\tau} .
$$

In particular, $w_{n} \rightarrow w$ in $L_{\mathrm{X}}^{1}\left(J_{\tau}\right)$ implies $u\left(\cdot ; \tau, x_{0}, w_{n}\right) \rightarrow u\left(\cdot ; \tau, x_{0}, w\right)$ in $C_{\mathrm{X}}\left(J_{\tau}\right)$. If $w \in L_{\mathrm{X}}^{1}(J)$ then $u\left(\cdot ; \tau, x_{0}, w\right)$ denotes $u\left(\cdot ; \tau, x_{0}, w_{\mid J_{\tau}}\right)$. With these notations, the semigroup property of solutions reads

$$
u\left(t ; \tau, x_{0}, w\right)=u\left(t ; \bar{\tau}, u\left(\bar{\tau} ; \tau, x_{0}, w\right), w\right) \quad \text { for all } 0 \leq \tau \leq \bar{\tau} \leq t \leq a .
$$

If $\tau=0$ and $x_{0}$ is fixed we simply write $u(\cdot ; w)$ instead of $u\left(\cdot ; \tau, x_{0}, w\right)$.

Let us also note that the autonomous problem, i.e. (4) with $w=0$, has a unique mild solution for every $x_{0} \in \overline{D(A)}$ if $A$ is accretive and satisfies the weak range condition

$$
\lim _{h \rightarrow 0+} h^{-1} \rho(x, R(I+h A))=0 \quad \text { for all } x \in \overline{D(A)} .
$$

In proofs of this result one point is to show that if $\left(x_{k}\right)_{k \geq 0}$ is a solution of the above implicit difference scheme such that $t_{k} \nearrow t_{\infty}<a$, then $x_{k} \rightarrow x_{\infty}$ for some $x_{\infty} \in \overline{D(A)}$; this fact will be used later on.

Proofs of all facts mentioned so far can be found in [2] or [4].

Finally, we shall need the following compactness result for mild solutions of (4), which is Theorem 2 in [1].

Lemma 1. Let $X$ be a real Banach space, $A: X \rightarrow 2^{X}$ be $m$-accretive such that $-A$ generates a compact semigroup and let $J=[0, a] \subset \mathbb{R}, W=\{w \in$ $L_{\mathrm{X}}^{1}(J):|w(t)| \leq \varphi(t)$ a.e. on $\left.J\right\}$ with $\varphi \in L^{1}(J)$. Then $\{u(\cdot ; w): w \in W\}$ is relatively compact in $C_{\mathrm{X}}(J)$.

In fact, the assertion of Lemma 1 remains true if $W$ is replaced by any uniformly integrable subset of $L_{\mathrm{X}}^{1}(J)$; see Theorem 2.3.2 in [23].

\section{Existence OF MILD SOLUTIONS UNDER TIME-DEPENDENT CONSTRAINTS}

Our main result concerning problem (1) is

Theorem 1. Let $X$ be a real Banach space and $A: D(A) \subset X \rightarrow 2^{X} \backslash \emptyset$ be $m$-accretive such that $-A$ generates a compact semigroup. Let $J=[0, a] \subset \mathbb{R}$ 
and $K: J \rightarrow 2^{X}$ be such that $K_{A}(0) \neq \emptyset$ and $g r\left(K_{A}\right)$ is closed from the left. Let $f: \operatorname{gr}\left(K_{A}\right) \rightarrow X$ be continuous, satisfying (2) and (3). Then

$$
u^{\prime}+A u \ni f(t, u) \quad \text { on } J, \quad u(0)=x_{0}
$$

has a mild solution for every $x_{0} \in K_{A}(0)$.

Proof. 1. To simplify subsequent arguments, we first reduce to the case when $f$ is bounded on $\operatorname{gr}\left(K_{A}\right)$. For this purpose, let $r(\cdot)$ be the solution of

$$
r^{\prime}(t)=1+c\left(1+r(t)+\left|S(t) x_{0}\right|\right) \quad \text { on } J, \quad r(0)=0,
$$

and define

$$
\hat{K}(t):=K(t) \cap \bar{B}_{r(t)}\left(S(t) x_{0}\right) \quad \text { and } \hat{K}_{A}(t):=\hat{K}(t) \cap \overline{D(A)} \text { for } t \in J .
$$

Evidently, $x_{0} \in \hat{K}_{A}(0)$, gr $\left(\hat{K}_{A}\right)$ is closed from the left and $f$ is bounded on $\operatorname{gr}\left(\hat{K}_{A}\right)$. In order to show that (2) also holds for $\hat{K}$ instead of $K$, let $t \in[0, a)$, $x \in \hat{K}_{A}(t)$ and $z:=f(t, x)$. Due to (2) there are sequences $h_{n} \rightarrow 0+$ and $e_{n} \rightarrow 0$ such that

$$
S_{z}\left(h_{n}\right) x+h_{n} e_{n} \in K_{A}\left(t+h_{n}\right) \text { for all } n \geq 1 .
$$

By means of the estimate

$$
\begin{gathered}
\left|S_{z}\left(h_{n}\right) x+h_{n} e_{n}-S\left(t+h_{n}\right) x_{0}\right| \leq \\
\left|S_{z}\left(h_{n}\right) x-S\left(h_{n}\right) x\right|+\left|x-S(t) x_{0}\right|+h_{n}\left|e_{n}\right| \leq \\
h_{n}|f(t, x)|+r(t)+h_{n}\left|e_{n}\right| \leq \\
r(t)+h_{n} c\left(1+r(t)+\left|S(t) x_{0}\right|\right)+h_{n}\left|e_{n}\right| \leq r\left(t+h_{n}\right),
\end{gathered}
$$

which holds if $n \geq 1$ is sufficiently large, this implies

$$
S_{z}\left(h_{n}\right) x+h_{n} e_{n} \in \hat{K}_{A}\left(t+h_{n}\right) \text { for all large } n \geq 1,
$$

hence (2) also holds for $\hat{K}$. Consequently, all assumptions of Theorem 1 are also satisfied if $K$ is replaced by $\hat{K}$, and we may therefore assume that $f$ is bounded on $\operatorname{gr}\left(K_{A}\right)$.

2. We now show which type of $\epsilon$-approximate solutions can be expected for (1), where we start with the usual exploitation of the subtangential condition. Fix $\epsilon \in(0,1]$. Since $z_{0}:=f\left(0, x_{0}\right) \in T_{K}^{A}\left(0, x_{0}\right)$, there is $h \in(0, \epsilon]$ such that $y_{1}:=S_{z_{0}}(h) x_{0}$ satisfies $\rho\left(y_{1}, K_{A}(h)\right) \leq \frac{1}{2} \epsilon h$, hence there is $x_{1} \in K_{A}(h)$ such that $\left|e_{0}\right| \leq \epsilon$ for $e_{0}:=\frac{x_{1}-y_{1}}{h}$. Then, letting $t_{0}=0$ and $t_{1}=t_{0}+h$,

$$
v(t):=S_{z_{0}}\left(t-t_{0}\right) x_{0}+\left(t-t_{0}\right) e_{0} \quad \text { on }\left[t_{0}, t_{1}\right]
$$

is a natural candidate as an approximate solution on $\left[t_{0}, t_{1}\right]$, and we may assume $\left|v(t)-x_{0}\right| \leq \epsilon$ on $\left[t_{0}, t_{1}\right]$ if $h>0$ is chosen small enough. Consequently, we get sequences $\left(t_{k}\right),\left(x_{k}\right),\left(z_{k}\right)$ and $\left(e_{k}\right)$ by induction such that

$$
\begin{gathered}
t_{k} \nearrow t_{\infty} \leq a, x_{k} \in K_{A}\left(t_{k}\right), z_{k}=f\left(t_{k}, x_{k}\right), \\
e_{k}=\left(x_{k+1}-S_{z_{k}}\left(t_{k+1}-t_{k}\right) x_{k}\right) /\left(t_{k+1}-t_{k}\right),\left|e_{k}\right| \leq \epsilon .
\end{gathered}
$$

For $k \geq 0$ we then let

$$
v(t)=S_{z_{k}}\left(t-t_{k}\right) x_{k}+\left(t-t_{k}\right) e_{k} \quad \text { on }\left[t_{k}, t_{k+1}\right],
$$


and may assume $t_{k+1}-t_{k} \leq \epsilon$ as well as $\left|v(t)-x_{k}\right| \leq \epsilon$ on $\left[t_{k}, t_{k+1}\right]$ by appropriate choice of the $t_{k}$. Of course $t_{\infty}<a$ is possible, and to be able to extend this approximate solution beyond $t_{\infty}$ we then need $\left(x_{k}\right)$ to be relatively compact.

To see that this is in fact true, let us first show

$$
\left|v(t)-u\left(t ; t_{k}, x_{k}, w\right)\right| \leq \epsilon\left(t-t_{k}\right) \quad \text { on }\left[t_{k}, t_{\infty}\right) \quad \text { for all } k \geq 0,
$$

where $w \in L_{\mathrm{X}}^{1}\left(\left[0, t_{\infty}\right]\right)$ is given by $w(t):=z_{k}$ on $\left[t_{k}, t_{k+1}\right)$; notice that $(7)$ in particular yields $|v(t)-u(t ; w)| \leq \epsilon t$ on $\left[0, t_{\infty}\right)$, hence

$$
w(t) \in f\left(\left[J_{t, \epsilon} \times \bar{B}_{\gamma \epsilon}(u(t ; w))\right] \cap \operatorname{gr}\left(K_{A}\right)\right) \text { a.e. on }\left[0, t_{\infty}\right]
$$

with $J_{t, \epsilon}=[t-\epsilon, t] \cap J$ and $\gamma=1+a$. Evidently, (7) holds if

$$
\left|v(t)-u\left(t ; t_{k}, x_{k}, w\right)\right| \leq \epsilon\left(t-t_{k}\right) \quad \text { on }\left[t_{j}, t_{j+1}\right]
$$

for all $j \geq k \geq 0$ and (9) is valid for $j=k$, by construction of $v$. Suppose that (9) holds for fixed $k \geq 0$ and $j=m-1 \geq k$. Exploitation of

$$
u\left(t ; t_{k}, x_{k}, w\right)=u\left(t ; t_{m}, u\left(t_{m} ; t_{k}, x_{k}, w\right), z_{m}\right) \quad \text { on }\left[t_{m}, t_{m+1}\right]
$$

and

$$
v(t)=u\left(t ; t_{m}, x_{m}, z_{m}\right)+\left(t-t_{m}\right) e_{m} \quad \text { on }\left[t_{m}, t_{m+1}\right]
$$

yields

$$
\begin{aligned}
\left|v(t)-u\left(t ; t_{k}, x_{k}, w\right)\right| & \leq\left|x_{m}-u\left(t_{m} ; t_{k}, x_{k}, w\right)\right|+\left(t-t_{m}\right)\left|e_{m}\right| \\
& \leq\left(t_{m}-t_{k}\right) \epsilon+\left(t-t_{m}\right) \epsilon
\end{aligned}
$$

for all $t \in\left[t_{m}, t_{m+1}\right]$, hence (9) holds for $j=m$. By induction (9) is therefore valid for all $j \geq k \geq 0$.

Now, relative compactness of $\left(x_{k}\right)=\left(v\left(t_{k}\right)\right)$ follows easily, since (7) implies

$$
v\left(\left[0, t_{\infty}\right)\right) \subset C_{k}+\left(t_{\infty}-t_{k}\right) \bar{B}_{\epsilon}(0) \quad \text { for all } k \geq 0,
$$

where $C_{k}:=v\left(\left[0, t_{k}\right]\right) \cup u\left(\left[t_{k}, t_{\infty}\right] ; t_{k}, x_{k}, w\right)$ is relatively compact. Evidently, this also yields relative compactness of $v\left(\left[0, t_{\infty}\right)\right)$.

Therefore, we may define $v\left(t_{\infty}\right):=\lim _{j \rightarrow \infty} x_{k_{j}}$, where $\left(x_{k_{j}}\right)$ is a convergent subsequence of $\left(x_{k}\right)$. Then it is easy to check that $(7)$ is still valid on $\left[t_{k}, t_{\infty}\right]$. Consequently, we are led to consider the set of approximate solutions defined by

$$
\begin{aligned}
M^{\epsilon} & =\{(v, w, P, b): b \in(0, a], \\
& v:[0, b] \rightarrow X \text { with } v(b) \in K_{A}(b), v([0, b]) \text { relatively compact, } \\
& w:[0, b] \rightarrow X \text { strongly measurable such that }(8) \text { holds a.e. on }[0, b], \\
& P \subset[0, b) \text { with } 0 \in P, b \in \bar{P} \text { such that } \tau \in P \text { implies } v(\tau) \in K_{A}(\tau) \\
& \text { and }|v(t)-u(t ; \tau, v(\tau), w)| \leq \epsilon(t-\tau) \text { on }[\tau, b]\} .
\end{aligned}
$$

3. By the arguments of step 2 we already know $M^{\epsilon} \neq \emptyset$, and we want to use Zorn's Lemma to obtain an element of $M^{\epsilon}$ with $b=a$. For this purpose we define a partial ordering on $M^{\epsilon}$ by $(v, w, P, b) \leq(\bar{v}, \bar{w}, \bar{P}, \bar{b})$ if

$$
b \leq \bar{b}, v=\bar{v} \text { on }[0, b], w=\bar{w} \text { a.e. on }[0, b], P \subset \bar{P} \text {. }
$$


To be able to apply Zorn's Lemma we have to show that every ordered subset $M \subset M^{\epsilon}$ has an upper bound in $M^{\epsilon}$. Let

$$
b^{*}=\sup \{b \in(0, a]:(v, w, P, b) \in M \text { for some } v, w, P\} .
$$

In case the "sup" is actually a "max", i.e. if there is $\left(v, w, P, b^{*}\right) \in M$, we let

$$
P^{*}=\left\{\tau \in\left[0, b^{*}\right): \text { there is }\left(v, w, P, b^{*}\right) \in M \text { with } \tau \in P\right\} .
$$

Evidently, $\left(v, w, P^{*}, b^{*}\right)$ is an upper bound and $\left(v, w, P^{*}, b^{*}\right) \in M^{\epsilon}$ is easy to check.

In the remaining case there is a sequence $\left(v_{n}, w_{n}, P_{n}, b_{n}\right) \subset M$ with $b_{n} \nearrow b^{*}$, hence $P_{n} \subset P_{n+1}, v_{n+1}=v_{n}$ on $\left[0, b_{n}\right]$ and $w_{n+1}=w_{n}$ a.e. on $\left[0, b_{n}\right]$ for all $n \geq 1$. We then let

$$
P^{*}=\bigcup_{n \geq 1} P_{n}, \quad v^{*}(t)=v_{n}(t) \text { on }\left[0, b_{n}\right], \quad w^{*}(t)=w_{n}(t) \text { on }\left[0, b_{n}\right] .
$$

Suppose for the moment that $v^{*}\left(\left[0, b^{*}\right)\right)$ is relatively compact. We then let $v^{*}\left(b^{*}\right)=\lim _{j \rightarrow \infty} v^{*}\left(b_{n_{j}}\right)$ where $\left(v^{*}\left(b_{n_{j}}\right)\right)$ is a convergent subsequence of $\left(v^{*}\left(b_{n}\right)\right)$, and claim that $\left(v^{*}, w^{*}, P^{*}, b^{*}\right) \in M^{\epsilon}$ is an upper bound for $M$. Evidently, $\left(v^{*}, w^{*}, P^{*}, b^{*}\right)$ is an upper bound for $M$, since $(v, w, P, b) \in M$ implies $b<b_{n}$, hence $(v, w, P, b) \leq\left(v_{n}, w_{n}, P_{n}, b_{n}\right)$ for some $n \geq 1$. To check that $\left(v^{*}, w^{*}, P^{*}, b^{*}\right) \in M^{\epsilon}$ is also easy; notice that $\tau \in P^{*}$ implies $\tau \in P_{n}$ and $v^{*}(\tau)=v_{n}(\tau)$ for all $n \geq n_{\tau}$. So, it remains to prove relative compactness of $v^{*}\left(\left[0, b^{*}\right)\right)$. But the latter follows by the corresponding arguments from step 2, where this time we take any sequence $\left(t_{k}\right) \subset P^{*}$ with $t_{k} \nearrow b^{*}$ and $x_{k}:=v^{*}\left(t_{k}\right)$; notice that (7) then holds with $v^{*}$ instead of $v$.

Consequently, there is a maximal element $\left(v^{*}, w^{*}, P^{*}, b^{*}\right) \in M^{\epsilon}$. Suppose $b^{*}<a$. We then let $t_{0}=b^{*}, x_{0}=v^{*}\left(b^{*}\right)$ and repeat the construction of step 2 to obtain the sequences from (5) and function $v$ from (6). Let

$$
\begin{gathered}
\bar{v}(t)=v^{*}(t) \text { on }\left[0, b^{*}\right], \quad \bar{v}(t)=v(t) \text { on }\left[b^{*}, t_{\infty}\right), \bar{b}=t_{\infty}, \\
\bar{w}(t)=w^{*}(t) \text { on }\left[0, b^{*}\right], \quad w^{*}(t)=z_{k} \text { on }\left[t_{k}, t_{k+1}\right], \bar{P}=P^{*} \cup\left\{t_{k}: k \geq 1\right\} .
\end{gathered}
$$

Then $v\left(\left[t_{0}, t_{\infty}\right)\right)$ is relatively compact again, and, as before, we let $\bar{v}\left(t_{\infty}\right):=$ $\lim _{j \rightarrow \infty} \bar{v}\left(t_{k_{j}}\right)$ for an appropriate subsequence $\left(t_{k_{j}}\right)$.

To obtain $(\bar{v}, \bar{w}, \bar{P}, \bar{b}) \in M^{\epsilon}$ we show that $\tau \in P^{*}$ and $t \in\left(\tau, t_{\infty}\right)$ implies $|\bar{v}(t)-u(t ; \tau, \bar{v}(\tau), w)| \leq \epsilon(t-\tau)$; the other cases as well as the remaining properties are rather obvious. Due to $(7)$ and the properties of $\left(v^{*}, w^{*}, P^{*}, b^{*}\right)$ we have

$$
\begin{gathered}
|\bar{v}(t)-u(t ; \tau, \bar{v}(\tau), w)| \leq \\
\left|v(t)-u\left(t ; t_{0}, x_{0}, w\right)\right|+\left|u\left(t ; t_{0}, x_{0}, w\right)-u\left(t ; t_{0}, u\left(t_{0} ; \tau, v^{*}(\tau), w\right), w\right)\right| \leq \\
\epsilon\left(t-t_{0}\right)+\left|v^{*}\left(t_{0}\right)-u\left(t_{0} ; \tau, v^{*}(\tau), w\right)\right| \leq \epsilon(t-\tau),
\end{gathered}
$$

hence $(\bar{v}, \bar{w}, \bar{P}, \bar{b}) \in M^{\epsilon}$ with $\bar{b}>b^{*}$, a contradiction. Consequently, $b^{*}=a$ for every maximal element of $M^{\epsilon}$.

4. Given $\epsilon_{m} \searrow 0$ there are $\left(v_{m}, w_{m}, P_{m}, a\right) \in M^{\epsilon_{m}}$ by steps 2 and 3 . Let $u_{m}=u\left(\cdot ; w_{m}\right)$. Since $\left|w_{m}(t)\right| \leq|f|_{\infty}$ a.e. on $J$ for all $m \geq 1$ and $S(\cdot)$ 
is compact the sequence $\left(u_{m}\right)$ is relatively compact in $C_{\mathrm{X}}(J)$ by Lemma 1 . W.l.o.g. $u_{m} \rightarrow u_{0}$ in $C_{\mathrm{X}}(J)$ and $u_{0}(0)=x_{0}$. For $t \in(0, a]$ there is $\left(t_{m}\right) \subset$ $[0, t]$ with $t_{m} \nearrow t$ sucht that $\rho\left(u_{m}\left(t_{m}\right), K_{A}\left(t_{m}\right)\right) \leq \epsilon_{m}$, hence $u_{m}\left(t_{m}\right) \rightarrow u_{0}(t)$ implies $u_{0}(t) \in K_{A}(t)$ since $\operatorname{gr}\left(K_{A}\right)$ is closed from the left. By (8), for almost all $t \in(0, a]$ we can choose a sequence $\left(t_{m}\right)$ such that $t_{m} \nearrow t$ and

$$
w_{m}(t) \in f\left(t_{m}, \bar{B}_{\gamma \epsilon_{m}}\left(u_{m}\left(t_{m}\right)\right) \cap K_{A}\left(t_{m}\right)\right) \quad \text { for all } m \geq 1,
$$

hence for every $\eta>0$ there is $m_{\eta} \geq 1$ such that

$$
w_{m}(t) \in f\left(t_{m}, \bar{B}_{\eta}\left(u_{0}\left(t_{m}\right)\right) \cap K_{A}\left(t_{m}\right)\right) \quad \text { for all } m \geq m_{\eta},
$$

and therefore $w_{m}(t) \rightarrow f\left(t, u_{0}(t)\right)$ a.e. on $J$. Consequently, $w_{m} \rightarrow f\left(\cdot, u_{0}(\cdot)\right)$ in $L_{\mathrm{X}}^{1}(J)$ which implies $u_{0}=\lim _{m \rightarrow \infty} u_{m}=u\left(\cdot ; f\left(\cdot, u_{0}(\cdot)\right)\right)$, i.e. $u_{0}$ is a mild solution of (1).

Let us note in passing that the necessary condition $K_{A}(t) \neq \emptyset$ on $J$ is of course implicitly contained in the assumptions of Theorem 1 . Nevertheless, we did not include this condition explicitly, since the reduction to bounded $f$ becomes easier this way.

Notice that compactness of the semigroup generated by $-A$ was only used in the final step to get relative compactness of $\left(u\left(\cdot ; w_{m}\right)\right)$ in $C_{\mathrm{X}}(J)$ via Lemma 1 . In the subsequent application to RD-systems the perturbation $f$ has the additional property that, restricted to $\operatorname{gr}\left(K_{A}\right)$,

(10) $f$ maps bounded sets into weakly relatively compact sets.

Since $B:=\left\{u_{m}(t): t \in J, m \geq 1\right\}+\bar{B}_{\gamma}(0)$ (with $u_{m}$ as in step 4 of the proof above $)$ is bounded and $w_{m}(t) \in f\left([J \times B] \cap \operatorname{gr}\left(K_{A}\right)\right)$ a.e. on $J$ for all $m \geq 1$, it follows from Corollary 2.6 in [13] that $\left(w_{m}\right)$ is weakly relatively compact in $L_{\mathrm{X}}^{1}(J)$. In this situation the proof of Theorem 1 obviously remains valid if $A$ is such that $w \rightarrow u(\cdot ; w)$ maps weakly relatively compact subsets of $L_{\mathrm{X}}^{1}(J)$ into relatively compact subsets of $C_{\mathrm{X}}(J)$. By the remark following Lemma 1 this property holds if $-A$ generates a compact semigroup. However, the former condition is weaker, in general, and will be useful later on.

Let us record this modification of Theorem 1 as

Theorem 2. Let $X$ be a real Banach space and $A: D(A) \subset X \rightarrow 2^{X} \backslash \emptyset$ be $m$-accretive such that $\{u(\cdot ; w): w \in W\}$ is relatively compact in $C_{\mathrm{X}}(J)$ for every fixed initial value in $\overline{D(A)}$ whenever $W \subset L_{\mathrm{X}}^{1}(J)$ is weakly relatively compact. Let $J=[0, a] \subset \mathbb{R}$ and $K: J \rightarrow 2^{X}$ be such that $K_{A}(0) \neq \emptyset$ and $\operatorname{gr}\left(K_{A}\right)$ is closed from the left. Let $f: \operatorname{gr}\left(K_{A}\right) \rightarrow X$ be continuous, satisfying (2), (3) and (10). Then

$$
u^{\prime}+A u \ni f(t, u) \quad \text { on } J, u(0)=x_{0}
$$

has a mild solution for every $x_{0} \in K_{A}(0)$.

In several applications it happens that for an appropriate choice of the $K(t)$ these sets are positively invariant for the resolvents of $A$. Then it is 
helpful to know that the subtangential condition can be separated, by which we mean that

$$
\begin{aligned}
& J_{\lambda} K(t) \subset K(t) \text { for } \lambda>0, t \in[0, a) \text { and } \\
& f(t, x) \in T_{K}(t, x) \text { for } t \in[0, a), x \in K_{A}(t)
\end{aligned}
$$

implies (2) if $\operatorname{gr}\left(K_{A}\right)$ is closed from the left. We do not have a simple direct proof of this fact, but it is not difficult to show that (11) implies the "weak range condition"

$$
\lim _{h \rightarrow 0+} h^{-1} \rho(x+h f(t, x),(I+h A)(K(t+h) \cap D(A)))=0
$$

$$
\text { for } t \in[0, a), x \in K_{A}(t) \text {, }
$$

and the latter in turn implies (2). This is the content of

Lemma 2. Let $X$ be a real Banach space and $A: D(A) \subset X \rightarrow 2^{X} \backslash \emptyset$ be $m$-accretive. Let $J=[0, a] \subset \mathbb{R}, K: J \rightarrow 2^{X}$ with $\operatorname{gr}\left(K_{A}\right)$ closed from the left and $f: \operatorname{gr}\left(K_{A}\right) \rightarrow X$ be continuous.

(a) Then (12) implies (2).

(b) Then (11) implies (2).

Proof. 1. To obtain (a), let $t_{0} \in[0, a)$ and $x_{0} \in K_{A}\left(t_{0}\right)$. Evidently, (2) holds if for every $\eta>0$ there is $\delta=\delta_{\eta} \in(0, \eta]$ such that

$$
\rho\left(S_{f\left(t_{0}, x_{0}\right)}(\delta) x_{0}, K_{A}\left(t_{0}+\delta\right)\right) \leq 3 \eta \delta .
$$

The idea is to construct local $\epsilon$-DS-approximate solutions for

$$
u^{\prime}+A u \ni f(t, u) \quad \text { on }\left[t_{0}, t_{0}+d\right], \quad u\left(t_{0}\right)=x_{0},
$$

and to compare them to corresponding $\epsilon$-DS-approximate solutions for

$$
v^{\prime}+A v \ni f\left(t_{0}, x_{0}\right) \quad \text { on }\left[t_{0}, t_{0}+d\right], \quad v\left(t_{0}\right)=x_{0} .
$$

Given $\eta \in(0,1]$, fix $r \in\left(0, a-t_{0}\right)$ such that $\left|f(t, x)-f\left(t_{0}, x_{0}\right)\right| \leq \eta$ for all $t \in\left[t_{0}, t_{0}+r\right], x \in \bar{B}_{r}\left(x_{0}\right) \cap K_{A}(t)$ and let $\epsilon \in(0, r)$ with $\epsilon \leq 1$. Exploitation of (12) yields $h_{k} \in(0, \epsilon]$ and $e_{k} \in X$ with $\left|e_{k}\right| \leq \epsilon$ such that

$$
x_{k+1}:=J_{h_{k}}\left(x_{k}+h_{k}\left(f\left(t_{k}, x_{k}\right)+e_{k}\right)\right) \in K_{A}\left(t_{k+1}\right) \text { for } k \geq 0
$$

where $t_{k+1}:=t_{k}+h_{k}$. Given these $h_{k}$ we also let

$$
\bar{x}_{k+1}:=J_{h_{k}}\left(\bar{x}_{k}+h_{k} f\left(t_{0}, x_{0}\right)\right) \quad \text { for } k \geq 0, \quad \bar{x}_{0}:=x_{0} .
$$

Since all $J_{h_{k}}$ are nonexpansive it follows by induction that

$$
\begin{gathered}
\left|x_{k}-\bar{x}_{k}\right| \leq\left(t_{k}-t_{0}\right)\left(\epsilon+\max _{j=1, \ldots, k-1}\left|f\left(t_{j}, x_{j}\right)-f\left(t_{0}, x_{0}\right)\right|\right), \\
\left|\bar{x}_{k}-x_{0}\right| \leq\left(t_{k}-t_{0}\right)\left|f\left(t_{0}, x_{0}\right)\right|+\left|J_{h_{k-1}} \cdots J_{h_{0}} x_{0}-x_{0}\right|,
\end{gathered}
$$

hence

$$
\left|x_{k}-x_{0}\right| \leq\left(t_{k}-t_{0}\right)\left(2+\left|f\left(t_{0}, x_{0}\right)\right|+|y|\right)+2\left|x_{0}-x\right|
$$

for all $(x, y) \in A$ as long as $t_{k}-t_{0} \leq r$ and $\left|x_{k}-x_{0}\right| \leq r$. Let $x \in D(A)$ with $\left|x_{0}-x\right| \leq r / 4, y \in A x$ and $d=\frac{1}{2} r\left(2+\left|f\left(t_{0}, x_{0}\right)\right|+|y|\right)^{-1}$, where we may assume $d \leq \eta$. Then (18) yields $\left|x_{k}-x_{0}\right| \leq r$ for all $k \geq 1$ such that $t_{k} \leq t_{0}+d$.

To obtain an $\epsilon$-DS-approximate solution for (13) from (15), we have to show 
that the $h_{k}$ can be chosen such that $t_{m} \geq t_{0}+d$ for some $m \geq 1$. This can be achieved by the usual trick: For $t \in[0, a)$ and $x \in K_{A}(t)$ let

$\varphi_{\epsilon}(t, x)=\sup \{h \in(0, \epsilon]: \rho(x+h f(t, x),(I+h A)(K(t+h) \cap D(A))) \leq \epsilon h\}$

and choose $h_{k} \geq \frac{1}{2} \varphi_{\epsilon}\left(t_{k}, x_{k}\right)$, say, in each step. Suppose $t_{k} \nearrow t_{\infty} \leq t_{0}+d$. Given $j \geq 0$ we then let $\bar{x}_{k}$ be given by (16), but starting at $k=j$ instead of $k=0$ (i.e., $\bar{x}_{j}=x_{j}$ ). Since (16) means $\bar{x}_{k+1}=J_{h_{k}}^{z} \bar{x}_{k}$ where $J_{\lambda}^{z}$ is the resolvent of $A_{z}$ with $z:=f\left(t_{0}, x_{0}\right)$, we know that $\left(\bar{x}_{k}\right)$ is a Cauchy sequence. Hence

$$
\left|x_{k+l}-x_{k}\right| \leq\left(t_{k+l}-t_{j}\right)(\epsilon+1)+\left(t_{k}-t_{j}\right)(\epsilon+1)+\left|\bar{x}_{k+l}-\bar{x}_{k}\right|
$$

for all $l \geq 1, k>j \geq 0$ shows that $\left(x_{k}\right)$ is a Cauchy sequence too. Consequently, $x_{k} \rightarrow x_{\infty} \in K_{A}\left(t_{\infty}\right)$ as $k \rightarrow \infty$ and therefore

$$
\lim _{(t, x) \rightarrow \overline{\left(t_{\infty}-, x_{\infty}\right)}} \varphi_{\epsilon}(t, x) \leq \lim _{k \rightarrow \infty} \varphi_{\epsilon}\left(t_{k}, x_{k}\right) \leq 2 \lim _{k \rightarrow \infty} h_{k}=0 .
$$

This is a contradiction, since we will show

$$
\lim _{(s, y) \rightarrow(t-, x)} \varphi_{\epsilon}(s, y)>0 \quad \text { for all } t \in[0, a), x \in K_{A}(t) .
$$

For this purpose, choose $h \geq \frac{1}{2} \varphi_{\epsilon / 3}(t, x)>0$ and $e \in B_{\epsilon / 2}(0)$ such that

$$
x+h(f(t, x)+e) \in(I+h A)(K(t+h) \cap D(A)) .
$$

Given $t_{n} \nearrow t$ and $x_{n} \in K_{A}\left(t_{n}\right)$ with $x_{n} \rightarrow x$, let $h_{n}=h+t-t_{n} \geq h$. Then

$$
J_{h}(x+h(f(t, x)+e)) \in K(t+h) \cap D(A)=K\left(t_{n}+h_{n}\right) \cap D(A) .
$$

Using the resolvent identity and letting $z:=x+h(f(t, x)+e)$, we get

$$
J_{h} z=J_{h_{n}}\left(z+\frac{t-t_{n}}{h}\left(z-J_{h} z\right)\right)
$$

hence

$$
z+\frac{t-t_{n}}{h}\left(z-J_{h} z\right) \in\left(I+h_{n} A\right)\left(K\left(t_{n}+h_{n}\right) \cap D(A)\right)=: R_{n}
$$

and therefore

$$
\begin{gathered}
\rho\left(x_{n}+h_{n} f\left(t_{n}, x_{n}\right), R_{n}\right) \leq\left|x-x_{n}\right|+h\left|f(t, x)-f\left(t_{n}, x_{n}\right)\right|+ \\
\left(t-t_{n}\right)\left(\left|f\left(t_{n}, x_{n}\right)\right|+\left|z-J_{h} z\right| / h\right)+\epsilon \frac{h}{2} \leq \epsilon h \leq \epsilon h_{n}
\end{gathered}
$$

for all large $n \geq 1$, i.e. $\lim _{n \rightarrow \infty} \varphi_{\epsilon}\left(t_{n}, x_{n}\right) \geq h>0$ and consequently (19) holds. Thus we get $\epsilon$-DS-approximate solutions $u^{\epsilon}, v^{\epsilon}$ for (13), (14) having the values $x_{k}, \bar{x}_{k}$ on $\left(t_{k-1}, t_{k}\right]$ for $k=1, \ldots, m$, respectively, and $t_{m}<t_{0}+d \leq$ $t_{m}+\epsilon$. Moreover, by (15) and (17),

$$
\begin{gathered}
\rho\left(\bar{x}_{k}, K_{A}\left(t_{k}\right)\right) \leq \\
\left(t_{k}-t_{0}\right)\left(\epsilon+\sup \left\{\left|f\left(t_{0}, x_{0}\right)-f(t, x)\right|: t \in\left[t_{0}, t_{0}+r\right], x \in K_{A}(t) \cap \bar{B}_{r}\left(x_{0}\right)\right\}\right)
\end{gathered}
$$

for $k=1, \ldots, m$, hence $\rho\left(\bar{x}_{k}, K_{A}\left(t_{k}\right)\right) \leq\left(t_{k}-t_{0}\right)(\epsilon+\eta)$. Given $\epsilon \rightarrow 0+$ we have $v^{\epsilon}(t) \rightarrow S_{f\left(t_{0}, x_{0}\right)}\left(t-t_{0}\right) x_{0}$ uniformly on $\left[t_{0}, t_{0}+d\right)$. Now notice that 
the choice of $d>0$ above was in fact independent of $\epsilon \in(0,1]$. Therefore, we find $\epsilon \in(0, \eta]$ such that $t_{m}-t_{0} \geq d-\epsilon \geq d / 2$ and

$$
\left|v^{\epsilon}(t)-S_{f\left(t_{0}, x_{0}\right)}\left(t-t_{0}\right) x_{0}\right| \leq \frac{1}{2} \eta d \quad \text { on }\left[t_{0}, t_{m}\right] .
$$

Let $\delta=t_{m}-t_{0}$. Then

$$
\rho\left(S_{f\left(t_{0}, x_{0}\right)}(\delta) x_{0}, K_{A}\left(t_{0}+\delta\right)\right) \leq \eta \delta+\rho\left(\bar{x}_{m}, K_{A}\left(t_{m}\right)\right) \leq 3 \eta \delta,
$$

hence $(2)$ holds.

2. In the situation of (b) let $t \in[0, a)$ and $x \in K_{A}(t)$. Then, given $\epsilon>0$, there is $h \in(0, \epsilon]$ and $e \in X$ with $|e| \leq \epsilon$ such that $x+h(f(t, x)+e) \in K(t+h)$, hence

$$
J_{h}(x+h(f(t, x)+e)) \in K(t+h) \cap D(A) .
$$

Consequently,

$$
\rho(x+h f(t, x),(I+h A)(K(t+h) \cap D(A))) \leq h \epsilon
$$

and therefore (12) holds. By step 1 of this proof the latter implies (2).

Theorem 1 and Theorem 2 together with Lemma 2 obviously imply

Corollary 1. Let $X$ be a real Banach space, $A: D(A) \subset X \rightarrow 2^{X} \backslash \emptyset$ be $m$-accretive, $J=[0, a] \subset \mathbb{R}, K: J \rightarrow 2^{X}$ with $K_{A}(0) \neq \emptyset$ and $g r\left(K_{A}\right)$ closed from the left. Let $f: \operatorname{gr}\left(K_{A}\right) \rightarrow X$ be continuous, satisfying (3). In addition, assume that $-A$ generates a compact semigroup, or $f$ satisfies (10) and $\{u(\cdot ; w): w \in W\}$ is relatively compact in $C_{\mathrm{X}}(J)$ for every fixed initial value in $\overline{D(A)}$ whenever $W \subset L_{\mathrm{X}}^{1}(J)$ is weakly relatively compact. Then

$$
u^{\prime}+A u \ni f(t, u) \quad \text { on } J, \quad u(0)=x_{0}
$$

has a mild solution for every $x_{0} \in K_{A}(0)$ if also (11) or (12) holds.

Additional information is contained in the following

Remarks. 1. In the situation of Theorem 1 but without the growth condition on $f$ we still get existence of a local solution of (1). This follows by application of Theorem 1 with $J$ and $K$ replaced by $\hat{J}=[0, b]$ and $\hat{K}: \hat{J} \rightarrow 2^{X}$ with $\hat{K}(t)=K(t) \cap \bar{B}_{t M}\left(S(t) x_{0}\right)$, respectively, where $b \in(0, a]$ and $M>1$ are chosen such that $|f(t, x)| \leq M-1$ on $\hat{J} \times \bar{B}_{r}\left(x_{0}\right)$ for $r:=b M+\max _{[0, b]}\left|S(t) x_{0}-x_{0}\right|$.

If $f$ is locally Lipschitz on $\operatorname{gr}\left(K_{A}\right)$, we may choose $\hat{J}$ and $\hat{K}$ above such that, in addition, $f$ is Lipschitz on $\operatorname{gr}\left(\hat{K}_{A}\right)$. Exploitation of the latter yields convergence of the $\epsilon_{m}$-approximate solutions $u_{m}$ from step 4 of the proof of Theorem 1, without using any compactness property of $A$. Evidently, this yields a local solution which can be extended up to a noncontinuable solution of (1). Moreover, this solution is unique. To summarize, problem (1) with $x_{0} \in K_{A}(0)$ has a unique noncontinuable solution if $A$ is $m$ accretive, $K: J=[0, a] \rightarrow 2^{X}$ is such that $\operatorname{gr}\left(K_{A}\right)$ is closed from the left and $f: \operatorname{gr}\left(K_{A}\right) \rightarrow X$ is locally Lipschitz, satisfying (2). 
2. Problem (1) has been considered in [22] in case $K(t) \equiv K$ is "semi locally closed". The "subtangential condition" used there is much stronger than (2): for closed $K$ it essentially becomes

$$
\lim _{h \rightarrow 0+} \sup \left\{h^{-1} \rho\left(S_{f(t, x)}(h) x, K\right):(t, x) \in J \times K\right\}=0 .
$$

Semilinear cases have been studied e.g. in [21], [19] and [6]. In the first paper the linear part $A$ is allowed to depend on time with varying domains $D(A(t))$ and existence of mild solutions is obtained under a necessary subtangential condition and a compactness assumption, either on the evolution system generated by the linear part or on the perturbation. In [19] multivalued perturbations are considered and existence of mild solutions is proven for compact semigroups under a strong subtangential condition. In [6], $\S 7$ it is shown that the latter result remains true under the necessary subtangential condition, and that the additional assumption on the semigroup can be replaced by a compactness assumption on the perturbation.

3. Let us note that for dissipative, not necessarily continuous $f: D(f) \subset$ $X \rightarrow X$ and $K(t) \equiv K$ the invariance results of [20] can be applied to $A-f$. In particular, in this situation Theorem 2 of [20] implies that for accretive $A$ problem (1) has a mild solution if for every $x \in K_{A}:=K \cap \overline{D(A)}$ and $\epsilon>0$ there is $h \in(0, \epsilon], x_{h} \in D(A) \cap D(f)$ and $y_{h} \in A x_{h}$ such that

$$
\left|x-x_{h}+h\left(f\left(x_{h}\right)-y_{h}\right)\right| \leq h \epsilon \quad \text { and } \quad \rho\left(x_{h}, K_{A}\right) \leq h \epsilon .
$$

In case $D(f)=K$ this is just the weak range condition for $A-f$, and it becomes (12) if, in addition, $f$ is continuous bounded and $\overline{K \cap D(A)}=K_{A}$.

\section{Application to Reaction-Diffusions-Systems: Global EXistence OF SOLUTIONS}

Let us start with the model problem

$$
u_{t}=\Delta \Phi(u)+g(u) \text { in }(0, \infty) \times \Omega, \quad \Phi(u(t, \cdot))_{\mid \partial \Omega}=0, \quad u(0, \cdot)=u_{0},
$$

where $\Omega \subset \mathbb{R}^{n}$ is open bounded with smooth boundary, $\Phi\left(u_{1}, \ldots, u_{m}\right)=$ $\left(\varphi_{1}\left(u_{1}\right), \ldots, \varphi_{m}\left(u_{m}\right)\right)$ with $\varphi_{k}: \mathbb{R} \rightarrow \mathbb{R}$ and $g: \mathbb{R}_{+}^{m} \rightarrow \mathbb{R}^{m}$.

To be able to apply the results from section 3 we need some information concerning the abstract formulation of the scalar nonlinear diffusion equation

$$
v_{t}=\Delta \varphi(v) \text { in }(0, T) \times \Omega, \quad \varphi(v(t, \cdot))_{\mid \partial \Omega}=0, \quad v(0, \cdot)=v_{0},
$$

where $\Omega$ is as above and $\varphi: \mathbb{R} \rightarrow \mathbb{R}$ is continuous increasing with $\varphi(0)=0$. Define $A: D(A) \subset L^{1}(\Omega) \rightarrow L^{1}(\Omega)$ by

$$
\begin{gathered}
A u=-\Delta \varphi(u), \\
D(A)=\left\{u \in L^{1}(\Omega): \varphi(u) \in W_{0}^{1,1}(\Omega), \Delta \varphi(u) \in L^{1}(\Omega)\right\} .
\end{gathered}
$$

Then (21) corresponds to the autonomous problem $u^{\prime}+A u \ni 0$. Let us collect some basic facts concerning $A$. Recall that $Q: L^{1}(\Omega) \rightarrow L^{1}(\Omega)$ is called order-preserving if $u \leq \bar{u}$ a.e. on $\Omega$ implies $Q u \leq Q \bar{u}$ a.e. on $\Omega$. 
Lemma 3. Let $\Omega \subset \mathbb{R}^{n}$ be open bounded with smooth boundary, $X=L^{1}(\Omega)$, $\varphi: \mathbb{R} \rightarrow \mathbb{R}$ be continuous increasing with $\varphi(0)=0$ and $A$ be given by (22). Then the following holds.

(a) $A$ is m-accretive with $\overline{D(A)}=X$.

(b) $J_{\lambda}: X \rightarrow X$ is order-preserving for all $\lambda>0$, and $J_{\lambda} u \leq|u|_{\infty}$ if $u \geq 0$.

(c) In addition, let $\varphi$ be strictly increasing. Then $\{u(\cdot ; w): w \in W\}$ is relatively compact in $C_{\mathrm{X}}(J)$ for every fixed initial value whenever $W \subset L_{\mathrm{X}}^{1}(J)$ is weakly relatively compact.

Assertion (a) and the first part of (b) are contained in Théorème 2.1 in [3], while the second part of (b) is a consequence of the same theorem combined with Corollaire 2.2. in [3]. Assertion (c) is Theorem 1 in [11].

To reformulate (20) as an abstract evolution system we let

$$
\begin{aligned}
& X=L^{1}(\Omega)^{m} \quad \text { with }|u|=\left|u_{1}\right|_{1}+\ldots+\left|u_{m}\right|_{1}, \\
& A u=-\Delta \Phi(u)=\left(-\Delta \varphi_{1}\left(u_{1}\right), \ldots,-\Delta \varphi_{m}\left(u_{m}\right)\right), \\
& D(A)=\left\{u \in X: \varphi_{k}\left(u_{k}\right) \in W_{0}^{1,1}(\Omega), \Delta \varphi_{k}\left(u_{k}\right) \in L^{1}(\Omega) \text { for } k=1, \ldots, m\right\}, \\
& f: D(f) \subset X^{+} \rightarrow X \text { defined by } f(u)(x)=g(u(x)) \text { on } \Omega,
\end{aligned}
$$

where $X^{+}=\left\{u \in X: u_{k} \geq 0\right.$ a.e. on $\Omega$ for $\left.k=1, \ldots, m\right\}$ is the positive cone in $X$ and $D(f)=\left\{u \in X^{+}: f(u) \in X\right\}$; notice that $L^{\infty}(\Omega)_{+}^{m} \subset D(f)$. Suppose that the $\varphi_{k}$ are continuous and strictly increasing with $\varphi_{k}(0)=0$. Then $A$ is $m$-accretive with $A(0)=0$, all $J_{\lambda}$ are order-preserving w.r. to the partial ordering induced by $X^{+}$on $X$ (i.e. $u \leq v$ if $v-u \in X^{+}$) and $J_{\lambda} u \leq u$ if $u(x)=\alpha \in \mathbb{R}_{+}^{m}$ a.e. on $\Omega$. This implies

$J_{\lambda} K \subset K \quad$ for all $\lambda>0$ and $K=\{u \in X: 0 \leq u \leq \bar{u}\}$ with $\bar{u} \equiv \alpha \in \mathbb{R}_{+}^{m}$,

i.e. such "rectangles" are positively invariant under $J_{\lambda}$. Moreover, due to Lemma $3(\mathrm{c})$ the operator $A$ satisfies the compactness assumption imposed in Corollary 1. Therefore, it is natural to look for "tubes" of type $C(t)=$ $[0, c(t)]$ with $c: \mathbb{R}_{+} \rightarrow \mathbb{R}_{+}^{m}$ such that gr $(C)$ is weakly positively invariant for $y^{\prime}=g(y)$. By Corollary 1 in [5] this holds if $g(y) \in T_{C}(t, y)$ for all $t \geq 0$, $y \in C(t)$. For this special $C(\cdot)$ the latter condition means

$$
\begin{array}{lll}
t \geq 0, y \in C(t) \text { with } y_{k}=0 & \text { implies } & g_{k}(y) \geq 0 \\
t \geq 0, y \in C(t) \text { with } y_{k}=c_{k}(t) & \text { implies } & g_{k}(y) \leq D^{+} c_{k}(t),
\end{array}
$$

where $D^{+}$denotes the upper right Dini derivative; see Chapter 9.1 in [6]. The first part of (23) is a natural assumption if $g$ models a chemical reaction, and to find an admissible upper bound $c(\cdot)$ we consider the initial value problem

$$
y^{\prime}=\hat{g}(y) \quad \text { on } \mathbb{R}_{+}, \quad y(0)=y_{0} \in \mathbb{R}_{+}^{m},
$$

with

$$
\hat{g}_{k}(y):=\max \left\{g_{k}(z): 0 \leq z \leq y, z_{k}=y_{k}\right\} ;
$$

notice that $g=\hat{g}$ on $\mathbb{R}_{+}^{m}$ iff $g$ is quasimonotone w.r. to $\mathbb{R}_{+}^{m}$. Let $\hat{y}\left(\cdot ; y_{0}\right)$ be any solution of $(24)$ with $[0, T)$ being its maximal interval of existence. Then $C(\cdot)=[0, \hat{y}(\cdot)]$ is weakly positively invariant for $y^{\prime}=g(y)$ on $[0, T)$. 
By application of Corollary 1 we therefore get

Theorem 3. Let $\Omega \subset \mathbb{R}^{n}$ be open bounded, $X=L^{p}(\Omega)^{m}$ with $m \geq 1$, $p \in[1, \infty)$ and $A: D(A) \subset X \rightarrow 2^{X} \backslash \emptyset$ be $m$-accretive with $0 \in A(0)$ such that all $J_{\lambda}$ are order-preserving with $J_{\lambda} u \leq u$ if $u \equiv \alpha \in \mathbb{R}_{+}^{m}$. Suppose also that $\{u(\cdot ; w): w \in W\}$ is relatively compact in $C_{\mathrm{X}}(J)$ for every fixed initial value in $\overline{D(A)}$ whenever $W \subset L_{\mathrm{X}}^{1}(J)$ is weakly relatively compact. Let $g$ : $\mathbb{R}_{+}^{m} \rightarrow \mathbb{R}^{m}$ be continuous with $g_{k}(y) \geq 0$ if $y_{k}=0$ and $f: D(f) \subset X^{+} \rightarrow X$ be defined by $f(u)(x)=g(u(x))$ on $\Omega$. Then the abstract RD-system

$$
u^{\prime}+A u \ni f(u) \quad \text { on } \mathbb{R}_{+}, \quad u(0)=u_{0}
$$

has a global mild solution in $X$ for $0 \leq u_{0} \in L^{\infty}(\Omega)^{m} \cap \overline{D(A)}$, if (24) with $\hat{g}$ from (25) has a global solution for $y_{0}=\left(\left|u_{0,1}\right|_{\infty}, \ldots,\left|u_{0, m}\right|_{\infty}\right)$.

Proof. Let $c(\cdot)$ denote the global solution of $(24), C(t):=[0, c(t)]$ and

$$
K(t):=\{u \in X: u(x) \in C(t) \text { a.e. on } \Omega\} .
$$

Notice first that $C(\cdot)$ is continuous w.r. to $d_{H}$ and bounded on $[0, a]$ for every $a>0$. Let $1 \leq p<\infty$. Then

$$
\rho(u, K(t))^{p}=\int_{\Omega} \rho(u(x), C(t))^{p} d x \quad \text { for every } u \in X,
$$

which follows from the fact that $P_{C(t)}(u(\cdot))$ has a measurable selection for every $u \in X$ by Proposition 3.2 in [10], where $P_{C(t)}$ denotes the metric projection onto $C(t)$.

To check that $\operatorname{gr}\left(K_{A}\right)$ is closed from the left, let $t_{k} \nearrow t$ and $u_{k} \in K_{A}\left(t_{k}\right)$ with $u_{k} \rightarrow u \in X$. Passing to an appropriate subsequence, we may assume $u_{k}(x) \rightarrow u(x)$ a.e. on $\Omega$ which implies $u(x) \in C(t)$ a.e. on $\Omega$, hence $u \in K_{A}(t)$. Since $g$ is continuous and bounded on $C([0, a])$ it follows that $f$ is continuous and bounded on $K([0, a])$ with $f(K([0, a])) \subset X$ weakly relatively compact. For $t \geq 0$ and $y \in C(t)$ with $y_{k}=c_{k}(t)$ we have $g_{k}(y) \leq \hat{g}_{k}(c(t))=c_{k}^{\prime}(t)$ by construction of $\hat{g}$. Since the first part of (23) holds by assumption, this implies $g(y) \in T_{C}(t, y)$. Due to $c_{k}^{\prime}(t)=D_{+} c_{k}(t)$ we even get

$$
\lim _{h \rightarrow 0+} h^{-1} \rho(y+h g(y), C(t+h))=0,
$$

hence

$$
h^{-1} \rho(u+h f(u), K(t+h))=\left(\int_{\Omega}\left[h^{-1} \rho(u(x)+h g(u(x)), C(t+h))\right]^{p} d x\right)^{1 / p}
$$

together with the dominated convergence theorem imply $f(u) \in T_{K}(t, u)$ for $t \geq 0, u \in K(t)$.

Evidently, $J_{\lambda} K(t) \subset K(t)$ for all $t \geq 0$ and $\lambda>0$. Therefore (1) with $f(t, u):=f(u)$ has a mild solution $u_{k}$ on $[0, k]$ for every $k \geq 1$, by Corollary 1 . Let $\left(u_{k_{j}}\right)$ be a subsequence of $\left(u_{k}\right)$ which converges uniformly on bounded intervals to a mild solution $u$ of (1). Then $u$ is a global mild solution of the abstract RD-system.

Due to Lemma 3 this result applies to the model problem (20). This yields 
Corollary 2. Let $\Omega \subset \mathbb{R}^{n}$ be open bounded with smooth boundary, and $\varphi_{k}: \mathbb{R} \rightarrow \mathbb{R}$ be continuous and strictly increasing with $\varphi_{k}(0)=0$ for $k=$ $1, \ldots, m$. Let $g: \mathbb{R}_{+}^{m} \rightarrow \mathbb{R}^{m}$ be continuous with $g_{k}(y) \geq 0$ if $y_{k}=0$. Then problem (20) has a global mild solution in $L^{1}(\Omega)^{m}$ for $0 \leq u_{0} \in L^{\infty}(\Omega)^{m}$, if (24) with $\hat{g}$ from (25) has a global solution for $y_{0}=\left(\left|u_{0,1}\right|_{\infty}, \ldots,\left|u_{0, m}\right|_{\infty}\right)$.

Remarks. 4. A similar invariance approach is used in [17] to obtain global existence for RD-systems with linear diffusion and smooth quasimonotone reaction terms.

In [16] the model problem (20) is considered for $m=2$, but with Dirichlet boundary conditions replaced by the mixed boundary conditions $\frac{\partial \varphi_{k}}{\partial \nu}\left(u_{k}\right)+$ $\alpha_{k} \varphi_{k}\left(u_{k}\right)=0$ in $(0, \infty) \times \partial \Omega$, where the $\alpha_{k}$ are sufficiently smooth nonnegative functions. In this paper weak solutions of (20) are obtained in the following situation: Either $\varphi_{k} \equiv 0$ or $\varphi_{k}(0)=\varphi_{k}^{\prime}(0)=0$ and $\varphi_{k}(r), \varphi_{k}^{\prime}(r), \varphi_{k}^{\prime \prime}(r)>$ 0 for $r>0$. Furthermore $g$ is assumed to be smooth and quasimonotone w.r. to $\mathbb{R}_{+}^{2}$ with $g(0)=0$ such that for every $y \in \mathbb{R}_{+}^{2}$ there is $\bar{y} \geq y$ with $g(\bar{y}) \leq 0$. Notice that the latter assumption on $g$ implies positive invariance of $[0, \bar{y}]$ for $y^{\prime}=g(y)=\hat{g}(y)$, hence every solution of (24) exists globally.

5. Under the assumptions imposed on $\varphi$ in Lemma 3(c) the semigroup generated by $-A$ (with $A$ from (22)) need not be compact, but compactness of the semigroup is guaranteed if, in addition, $\varphi$ is continuously differentiable on $\mathbb{R} \backslash\{0\}$ such that $\varphi^{\prime}(r) \geq c|r|^{\gamma-1}$ on $\mathbb{R} \backslash\{0\}$ with some $c>0$ and $\gamma>\max \left\{0, \frac{n-2}{n}\right\}$; see Lemma 2.6.2 in [23].

In the special case $\varphi(r)=|r|^{\gamma-1} r$, which corresponds to the porous medium equation, the condition $\gamma>\max \left\{0, \frac{n-2}{n}\right\}$ is optimal in the sense that the semigroup is not compact for $0<\gamma<\frac{n-2}{n}$. This is a consequence of Theorem 8 in [8]; see Remark 11 there.

6. In chemical applications $g(\cdot)$ will usually be of special type (see e.g. [14]). In the simplest case $m=2$ a typical reaction term, corresponding to the chemical reaction $\alpha A+\beta B \rightarrow P$, is given by the so-called Freundlichs kinetics

$$
g(y)=\left(-\alpha k y_{1}^{\alpha} y_{2}^{\beta},-\beta k y_{1}^{\alpha} y_{2}^{\beta}\right) \quad \text { with } \alpha, \beta, k>0
$$

here $\alpha$, respectively $\beta$ is the order of the reaction w.r. to $A$, respectively $B$ and $k$ is the rate constant. Evidently $\hat{g}(y)=0$ on $\mathbb{R}_{+}^{2}$, hence (24) has global solutions for every $\alpha, \beta>0$.

In case of a mixed order reversible reaction $\alpha A \rightleftharpoons \beta B$ one has

$$
g(y)=\left(\alpha\left(k_{2} y_{2}^{\beta}-k_{1} y_{1}^{\alpha}\right), \beta\left(k_{1} y_{1}^{\alpha}-k_{2} y_{2}^{\beta}\right)\right) \quad \text { with } \alpha, \beta, k_{1}, k_{2}>0 .
$$

Here $g$ is quasimonotone with $g\left(k_{1}^{-1 / \alpha} r^{\beta}, k_{2}^{-1 / \beta} r^{\alpha}\right)=0$ for all $r>0$, hence (24) has global solutions for every $\alpha, \beta>0$.

Finally, if $g$ is given by

$$
g(y)=\left(-y_{1}^{\alpha} y_{2}^{\beta}, y_{1}^{\alpha} y_{2}^{\beta}\right) \quad \text { with } \alpha, \beta>0,
$$


we get $\hat{g}(y)=\left(0, y_{1}^{\alpha} y_{2}^{\beta}\right)$, hence $(24)$ has global solutions provided $\beta \leq 1$. Here as well as in the first example the use of Theorem 3, say, is to provide existence of solutions in cases when $g$ is not locally Lipschitz, rather than to obtain global existence.

In case of linear diffusion, more precisely if $\varphi_{k}(r)=d_{k} r$ with $d_{k}>0$ for $k=1,2$, existence of global solutions of (20) with $g$ as in the last example above and $u_{0} \in L^{\infty}(\Omega)^{2}$ with $u_{0} \geq 0$ was established in [18] for $\alpha=1$ and arbitrary $\beta \geq 1$ by different techniques; see also [15].

Additional difficulties occur e.g. in the case of so-called zero-order reactions, for instance $g$ from one of the examples above in the limit case $\alpha=0$, for the reaction term is then discontinuous. In this situation it is appropriate to replace $g$ by a certain multivalued "regularization" $G$. Corresponding RDsystems of type (20) have been considered recently in [12], where existence of local mild solutions was proven; see also Chapter 3.4 in [23] and [7].

Acknowledgment. Part of this work was done while the author was visiting the Université de Franche-Comté (Besançon). The author wishes to express his thanks to his colleagues of the Equipe de Mathématiques de Besançon for their kind hospitality. In particular, he is indebted to Professor Ph. Bénilan for helpful discussions.

\section{REFERENCES}

[1] P. Baras, Compacité de l'opérateur $f \rightarrow u$ solution d'une équation non linéaire $(d u / d t)+A u \ni f$, C. R. Acad. Sci. Paris Sér. A-B, 286 (1978), A1113-A1116.

[2] V. Barbu, Nonlinear Semigroups and Differential Equations in Banach Spaces, Noordhoff, Leyden, 1976.

[3] P. Bénilan, Équations d'évolution dans un espace de Banach quelconque et applications, Thèse d'Etat, Orsay, 1972.

[4] P. Bénilan, M. G. Crandall and A. Pazy, Nonlinear Evolution Equations in Banach Spaces, (monograph in preparation).

[5] D. Bothe, Multivalued differential equations on graphs, Nonl. Anal. 18 (1992), 245252 .

[6] D. Bothe, Multivalued differential equations on graphs and applications, Ph.D. Dissert., Paderborn, 1993.

[7] D. Bothe, Reaction diffusion systems with discontinuities. A viability approach, (to appear).

[8] H. Brézis and A. Friedman, Nonlinear parabolic equations involving measures as initial conditions, J. Math. Pures Appl. 62 (1983), 73-97.

[9] K. Deimling, Nonlinear Functional Analysis, Springer-Verlag, Berlin-New York, 1985.

[10] K. Deimling, Multivalued Differential Equations, De Gruyter, 1992.

[11] J. I. Diaz and I. I. Vrabie, Propriétés de compacité de l'opérateur de Green généralisé pour l'équation des milieux poreux, C. R. Acad. Sci. Paris Sér. I Math. 309 (1989), 221-223.

[12] J. I. Diaz and I. I. Vrabie, Existence for reaction diffusion systems. A compactness method approach, J. Math. Anal. Appl. 188 (1994), 521-540.

[13] J. Diestel, W. M. Ruess and W. Schachermayer, Weak compactness in $L^{1}(\mu, X)$, Proc. Amer. Math. Soc. 118 (1993), 447-453.

[14] P. Erdi and J. Toth, Mathematical Models of Chemical Reactions, Manchester Univ. Press, 1989. 
[15] S. L. Hollis, R. H. Martin and M. Pierre, Global existence and boundedness in reactiondiffusion systems, SIAM J. Math. Anal. 18 (1987), 744-761.

[16] L. Maddalena, Existence, uniqueness and qualitative properties of the solution of a degenerate nonlinear parabolic system, J. Math. Anal. Appl. 127 (1987), 443-458.

[17] R. H. Martin, Global existence questions for reaction-diffusion systems, in Semigroups, Theory and Applications (eds. Brézis, Crandall, Kappel), Pitman Res. Notes, 141, Longman, 1986, pp. 169-177.

[18] K. Masuda, On the global existence and asymptotic behavior of solutions of reactiondiffusion equations, Hokkaido Math. J. 12 (1983), 360-370.

[19] N. Pavel, Differential Equations, Flow Invariance and Applications, Res. Notes Math. 113, Pitman, 1984.

[20] M. Pierre, Invariant closed subsets for nonlinear semigroups, Nonl. Anal. 2 (1978), 107-117.

[21] J. Prüss, On semilinear parabolic evolution equations on closed sets, J. Math. Anal. Appl. 77 (1980), 513-538.

[22] I. I. Vrabie, Compactness methods and flow-invariance for perturbed nonlinear semigroups, Anal. Stiin. Univ. Iasi, 27 (1981), 117-124.

[23] I. I. Vrabie, Compactness Methods for Nonlinear Equations, Pitman, 1987.

FACHBEREICH 17

UNIVERSITÄT PADERBORN

D-33095 PAdERBorn, GERMANY

E-mail address: dieterb@uni-paderborn.de 


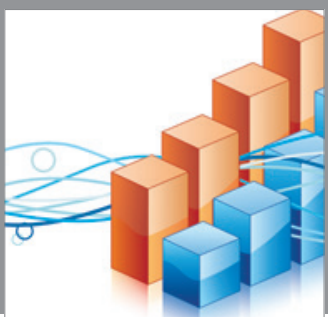

Advances in

Operations Research

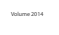

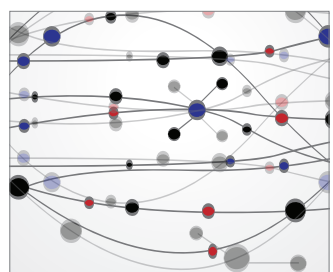

\section{The Scientific} World Journal
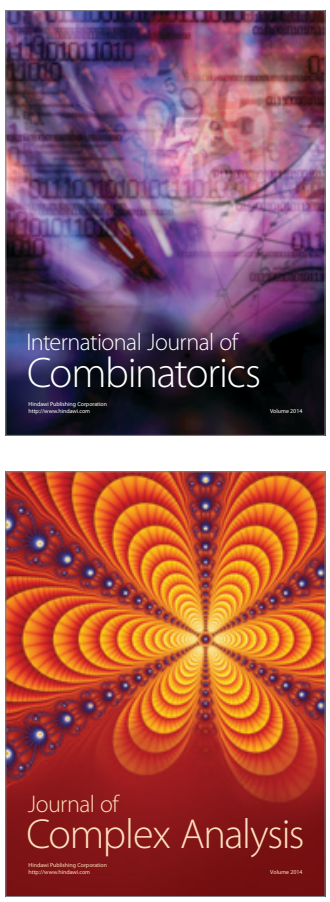

International Journal of

Mathematics and

Mathematical

Sciences
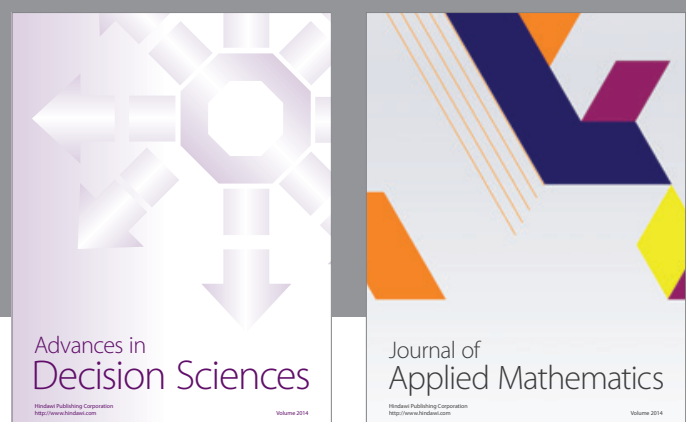

Journal of

Applied Mathematics
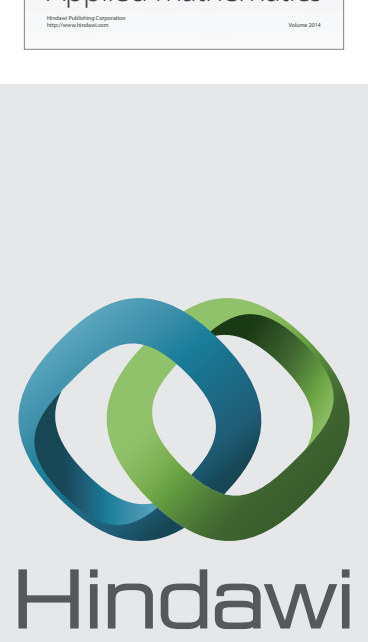

Submit your manuscripts at http://www.hindawi.com
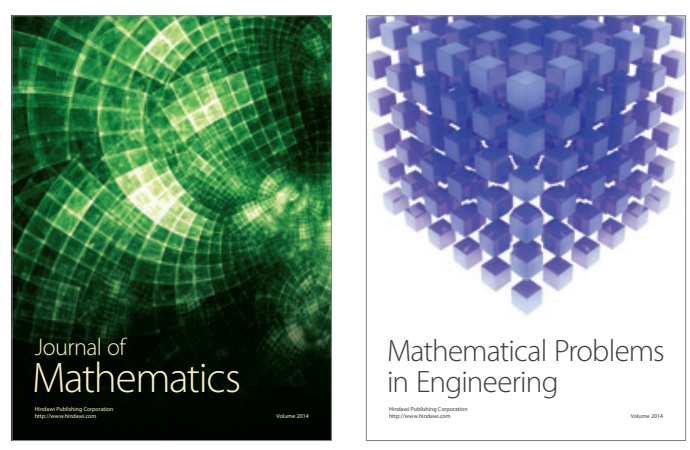

Mathematical Problems in Engineering
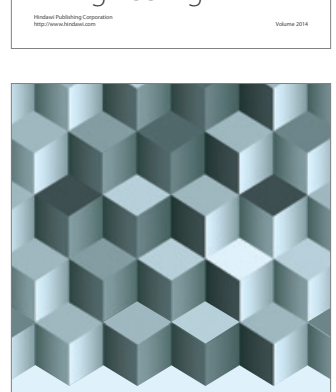

Journal of

Function Spaces
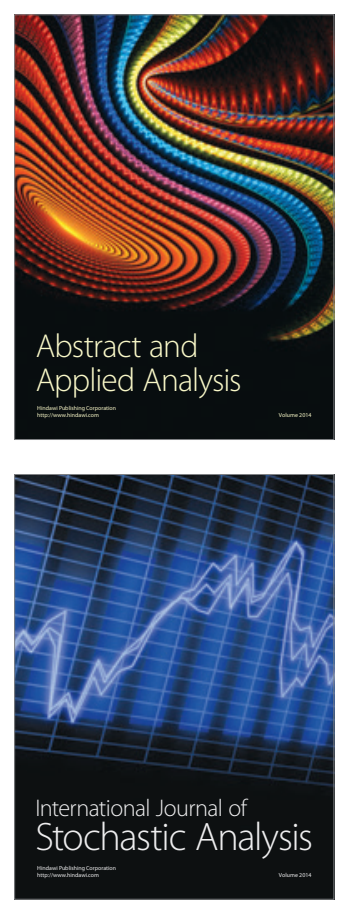

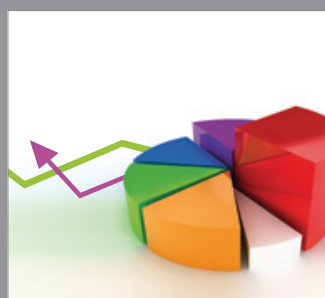

ournal of

Probability and Statistics

Promensencen
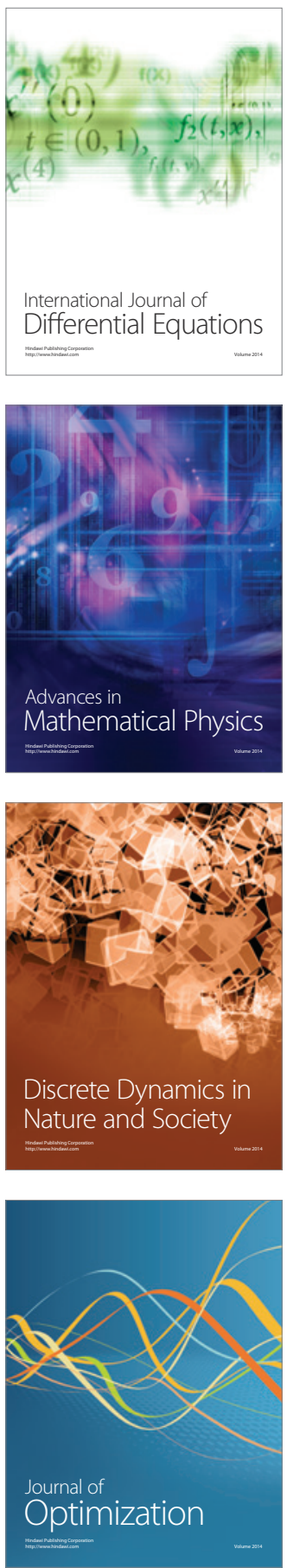